\title{
Angioplasty for Intracranial Vertebrobasilar Stenosis: A Meta-Analysis of Procedural Risks
}

Sepideh Amin-Hanjani MD1, Yi-Fan Chen $\mathrm{PhD}^{2}$, Hussain Shallwani $\mathrm{MD}^{3}$, Tanya Turan $\mathrm{MD}^{4}$,

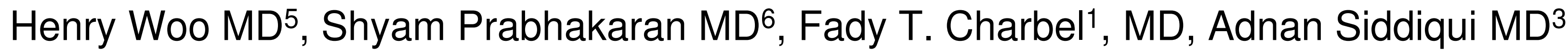

1Department of Neurosurgery, Univ of Illinois at Chicago, Chicago, IL

${ }^{2}$ Biostatistics Core, Univ of Illinois at Chicago, Chicago, IL

${ }^{3}$ Department of Neurosurgery, Univ of Buffalo, Buffalo, NY

${ }^{4}$ Department of Neurology, Medical Univ of South Carolina, Charleston, SC

${ }^{5}$ Department of Neurosurgery, Northwell Health, Long Island, NY

DEPARTMENT OF

${ }^{6}$ Department of Neurology, Northwestern Univ School of Medicine, Chicago, IL

Background: Symptomatic flow-compromised intracranial vertebrobasilar (VB) stenosis carries a significant risk of stroke ${ }^{1}$. Although flow restoration through endovascular interventions represents the most plausible treatment strategy, the high periprocedural risk of stenting, especially for the basilar artery, has proven prohibitive. Submaximal angioplasty alone may represent a safer strategy. We examined the periprocedural risks associated with VB submaximal angioplasty.

Methods: All English language studies of intracranial angioplasty for atherosclerotic disease were screened. Studies were included in the meta-analysis based on the following criteria: submaximal angioplasty alone, identifiable periprocedural outcomes for VB angioplasty, $\geq 5$ patients. Our primary outcome was the 30 day stroke/ death event rate. We used $Q$ statistics to test heterogeneity, and random-effect models using DerSimonian-Laird estimator to summarize effect sizes. Funnel plots and regression tests were performed to detect publication bias. We also examined the event rate difference between vertebral artery (VA) vs. basilar artery (BA).

Results: Six studies, comprising 127 patients, met inclusion criteria. The $Q$ test $(p=0.90)$ indicated homogeneity across studies. The pooled event rate was 5.9\% (95\% Cl, 2.9\%- 8.9\%). The funnel plot and a non-significant regression test $(p=0.30)$ indicated no publication bias. Examination for event rate of vertebral vs basilar angioplasty showed no statistically significant risk difference $(5 \%, 95 \% \mathrm{Ci}-1 \%-10 \%, \mathrm{p}=0.12)$.
Conclusions: Submaximal angioplasty represents a potentially promising intervention for treatment of symptomatic intracranial VB stenosis, and does not appear to prose higher risk in BA compared to intracranial VA stenosis.. These results support the need to prospectively examine the safety and feasibility of the intervention in flow-compromised symptomatic VB stenosis patients at high risk for recurrent stroke.

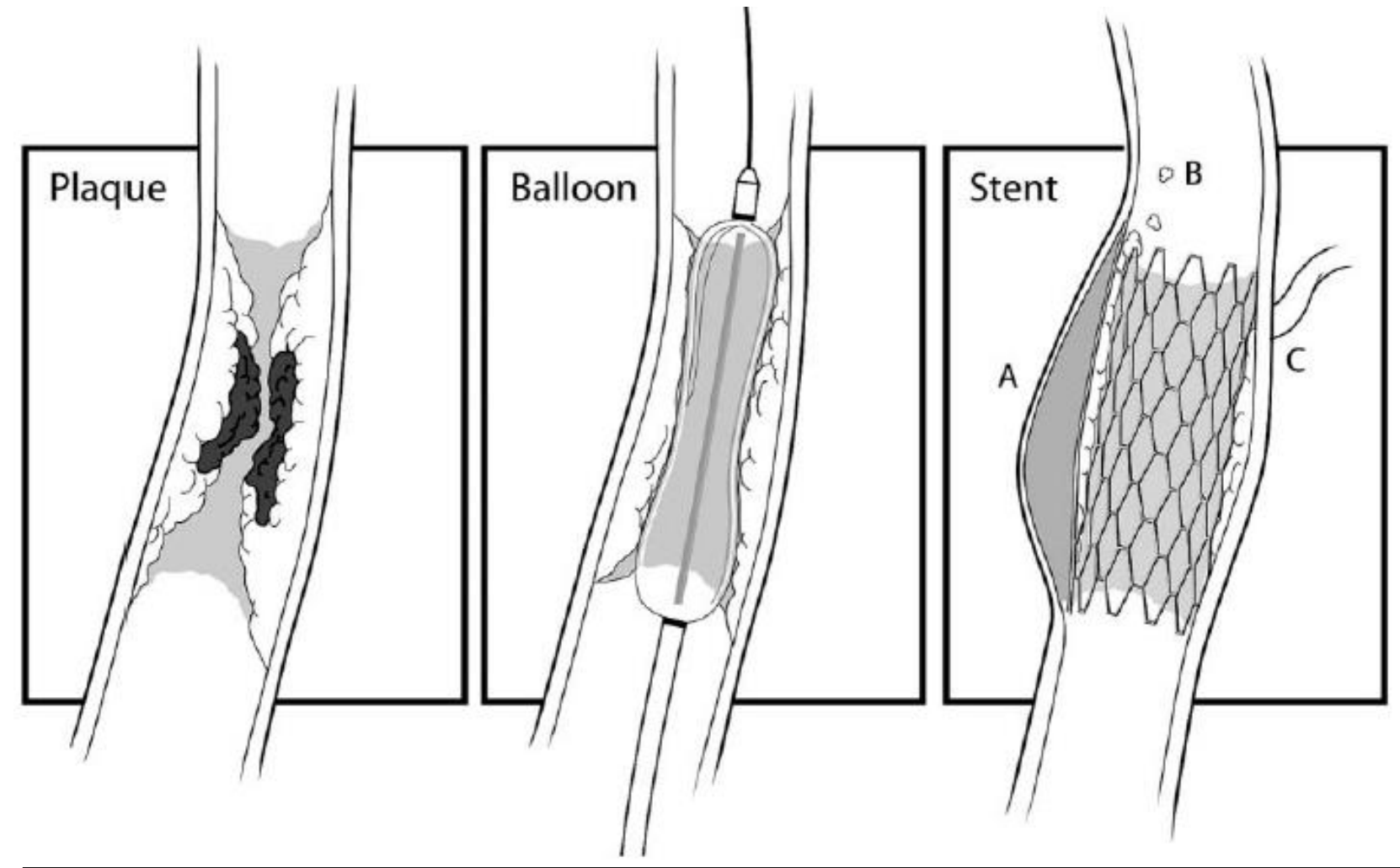

Schematic depiction of the difference between primary stenting and submaximal angioplasty (from Dumont et al, 2012²):

Submaximal balloon angioplasty results in vessel revascularization while limiting risk of perioperative events. Stenting, on the other hand, is more likely to result in A. trauma to the vessel wall causing dissection or perforation, B. plaque trauma, C. 'snowplow' effect of plaque into adjacent perforator vessels.

Forest plot for periprocedural event rate (30 day stroke/ death)

\begin{tabular}{|llllll}
\hline Author(s), Year & Event & No. subjects & Event proportion [95\% Cl] \\
\hline Marks et al, 2005 & 1 & 21 & $0.05[-0.04,0.14]$ \\
Wojak et al, 2006 & 3 & 40 & $0.07[-0.01,0.16]$ \\
Al-Ali et al, 2011 & 1 & 28 & $0.04[-0.03,0.10]$ \\
Nguyen et al, 2011 & 3 & 24 & $0.12[-0.01,0.26]$ \\
Dumont et al, 2012 & 0 & 8 & $0.06[-0.09,0.21]$ \\
Dumont et al, 2016 & 0 & 6 & & $0.07[-0.12,0.26]$ \\
\hline RE Model & & & 0.15 & $0.06[0.03,0.09]$ \\
\end{tabular}

